\title{
COVID-19 Disease Course in Former Smokers, Smokers and COPD Patients
}

\author{
Ricardo Wesley Alberca ${ }^{1 *}$, Júlia Cataldo Lima1 ${ }^{1}$ Emily Araujo de Oliveira1,2, \\ Sarah Cristina Gozzi-Silva 1,2, Yasmim Álefe Leuzzi Ramos', \\ Milena Mary de Souza Andrade' ${ }^{\text {, Danielle Rosa Beserra1, }}$ \\ Luana de Mendonça Oliveira1,2, Anna Cláudia Calvielli Castelo Branco ${ }^{1,2}$, \\ Anna Julia Pietrobon ${ }^{1,2}$, Nátalli Zanete Pereira1 ${ }^{1}$, Franciane Mouradian Emidio Teixeira ${ }^{1,2}$, \\ lara Grigoletto Fernandes ${ }^{1}$, Alberto José da Silva Duarte ${ }^{1}$, Gil Benard ${ }^{1}$ and \\ Maria Notomi Sato ${ }^{1 *}$

\section{'Laboratorio de Dermatologia e Imunodeficiencias (LIM-56), Departamento de Dermatologia, Faculdade de Medicina} \\ FMUSP, Universidade de São Paulo, São Paulo, Brazil, ${ }^{2}$ Institute of Biomedical Sciences, University of São Paulo, \\ São Paulo, Brazil
}

OPEN ACCESS

Edited by:

Diane S. Allen-Gipson, University of South Florida, United States

Reviewed by: Talha Bin Emran,

Begum Gulchemonara Trust

University, Bangladesh Todd A. Wyatt,

University of Nebraska Medical

Center, United States

*Correspondence:

Ricardo Wesley Alberca ricardowesley@gmail.com

Maria Notomi Sato marisato@usp.br

Specialty section:

This article was submitted to Integrative Physiology, a section of the journal

Frontiers in Physiology

Received: 04 December 2020 Accepted: 28 December 2020 Published: 27 January 2021

Citation:

Alberca RW, Lima JC, Oliveira EA, Gozzi-Silva SC, Ramos YÁL, Andrade MMS, Beserra DR, Oliveira LM, Branco ACCC, Pietrobon AJ, Pereira NZ, Teixeira FME, Fernandes IG, Duarte AGS, Benard G and Sato MN (2021) COVID-19 Disease Course in Former Smokers, Smokers and COPD Patients.

Front. Physiol. 11:637627. doi: 10.3389/fphys.2020.637627
The severe respiratory and systemic disease named coronavirus disease-2019 (COVID19 ) is caused by the severe acute respiratory syndrome coronavirus 2 (SARS-CoV-2). Currently, the COVID-19 pandemic presents a huge social and health challenge worldwide. Many different risk factors are associated with disease severity, such as systemic arterial hypertension, diabetes mellitus, obesity, older age, and other coinfections. Other respiratory diseases such as chronic obstructive pulmonary disease (COPD) and smoking are common comorbidities worldwide. Previous investigations have identified among COVID-19 patients smokers and COPD patients, but recent investigations have questioned the higher risk among these populations. Nevertheless, previous reports failed to isolate smokers and COPD patients without other comorbidities. We performed a longitudinal evaluation of the disease course of smokers, former smokers, and COPD patients with COVID-19 without other comorbidities, from hospitalization to hospital discharge. Although no difference between groups was observed during hospital admission, smokers and COPD patients presented an increase in COVID-19-associated inflammatory markers during the disease course in comparison to non-smokers and former smokers. Our results demonstrated that smoking and COPD are risk factors for severe COVID-19 with possible implications for the ongoing pandemic.

Keywords: SARS-CoV-2, COVID-19, infection, smoking, chronic obstructive pulmonary disease

\section{INTRODUCTION}

Chronic obstructive pulmonary disease (COPD) is a major public health problem, affecting millions worldwide. Common clinical symptoms are dyspnea, cough, and sputum production. The main risk factor for the development of COPD is tobacco smoking, which leads to pulmonary remodeling and inflammation (Marsh et al., 2006). Smoking and COPD are independent risk factors for other diseases such as lung cancer (Biondini et al., 2019) and respiratory infections (Gilca et al., 2011; Aikphaibul et al., 2020; Yoon et al., 2020). 
The infection caused by SARS-CoV-2 can lead to the development of a severe pulmonary and systemic disease named COVID-19 (Alberca et al., 2020c). Previous reports have identified that COVID-19 is often more severe in the elderly and individuals with comorbidities such as obesity, hypertension, diabetes Mellitus, other co-infections (Alberca, 2020; Alberca et al., 2020a,d,f; Castelo Branco et al., 2020; Shaw et al., 2020). Those patients generally present an increase in COVID-19associated inflammatory markers, such as d-dimer, leukocytes count, neutrophil count, neutrophil-to-lymphocyte (NTL) ratio, alanine aminotransferase (ALT), aspartate aminotransferase (AST), and c-reactive protein (CRP) (Wolff et al., 2020). It still not clear the mechanisms that could increase the susceptibility of those groups to a more severe COVID-19, but several mechanisms have been postulated such as dysregulation of the anti-viral immune response (Codo et al., 2020) and increase in the expression of the angiotensin-converting enzyme 2 (ACE2) receptor, SARS-CoV-2 entry receptor (Leung et al., 2020).

Nicotine, a component in tobacco smoke, can suppress antiviral immune responses, via downregulation of Interferon regulatory factor 7 (Han et al., 2019). COPD patients with frequent exacerbations also present a reduction in antiviral immune response, via a reduction in type I and III interferons and interferon-stimulated genes (Singanayagam et al., 2019). In addition, smokers and COPD patients also present an increase in ACE2 receptor expression in the lungs (Leung et al., 2020).

In one of the first reports with COVID-19 patients, $1.1 \%$ of the patients had COPD, $12.6 \%$ were smokers and $1.9 \%$ were former smokers (Guan et al., 2020). COPD and smoking have also been associated with an increased incidence in COVID-19 (Dai et al., 2020; Lian et al., 2020; Zhao et al., 2020) and worst prognoses (Barrasa et al., 2020; Patanavanich and Glantz, 2020).

However, a recent report identified that smoking and pulmonary diseases, such as COPD, were less common in COVID-19 in comparison to influenza patients (Auvinen et al., 2020) and one meta-analyses found no association between COVID-19 prognosis and smoking (Vardavas and Nikitara, 2020).

Nevertheless, to the moment, no study was performed to investigate the difference in the disease course of COVID-19 among smokers, former smokers, and COPD patients without other comorbidities (Dai et al., 2020). Therefore, we aimed to perform an investigation in our cohort to assess if smoking or COPD could influence the COVID-19 disease course.

\section{MATERIALS AND METHODS}

Patients were recruited at the university hospital (Hospital das Clinicas da Universidade de São Paulo - HCFMUSP), in a special ward for COVID-19 patients. Inclusion criteria were: (1) All patients have SARS-CoV-2 infection verified by nasopharyngeal detection of SARS-CoV-2 by reverse-transcriptase polymerase chain reaction; (2) COPD patients were selected based on previous COPD diagnoses, all patients in our cohort were considered mild to moderate COPD patients; (3) Smoking habits were self-reported by patients on the hospital's admission.
Exclusion criteria were: (1) any other comorbidities other than smoking or COPD; (2) Other co-infection (bacterial or viral). We tracked patients' clinical laboratory data performed from day 1 at the hospital until SARS-CoV-2 clearance and hospital discharge. This study was approved by the Ethics Committee of HCFMUSP (no. 30800520.7.0000.0068-2020).

In our cohort of 318 patients from the university hospital: Six patients were previously diagnosed with COPD (COPD), seven were active smokers (SMOKERS), and 12 have a previous smoking history (with over 10 years absence) without COPD (EX-SMOKERS), and 10 healthy individuals non-smokers (NC). No patients included in this investigation presented any other comorbidities. Data are shown for the longitudinal graph as median values. Data from the first hospitalization day are shown as median and standard error mean (SEM). Statistical analysis for survival curve was performed using Log-rank test, with Logrank test for trend and Gehan-Breslow-Wilcoxon test to compare all groups, statistical analysis for other data was performed with Kruskal-Wallis test with Dunn's multiple comparisons with GraphPad Prism 8 software (GraphPad Software, Inc., San Diego, CA, United States).

\section{RESULTS AND DISCUSSION}

The patients did not present any difference in age and hospitalization time was only increased in EX-SMOKERS in comparison to NC (Table 1). On the first hospitalization day, no difference was observed in inflammatory hallmarks of SARS-CoV-2 infection such as lactate dehydrogenase, C-reactive protein, alanine aminotransferase, aspartate aminotransferase, D-dimer, and alkaline phosphatase (Table 1; Alberca et al., 2020b). Previous reports have identified models for predicting the disease outcome based on the first day after hospitalization or based on a risk score (Yildiz et al., 2020). Nevertheless, we hypothesize that longitudinal analysis of clinical data is crucial for the understanding of the immune response to SARS-CoV2 , mainly because it is almost impossible to precisely determine the infection day. Therefore, we performed a daily comparison of clinical data for these patients.

During the disease course, no difference in the number of leukocytes was verified among groups (Figure 1A). The NTL ratio was increased in the EX-SMOKERS, SMOKERS, and COPD patients in comparison to NC. It is noteworthy that SMOKERS had a further increase in the NTL in relation to EX-SMOKERS and COPD (Figure 1B). NTL is a widely used maker for COVID19 prognoses (Alberca et al., 2020b), but the increase in NTL in the SMOKERS groups was due to both an increase in the neutrophil count (Figure 1C) in comparison to EX-SMOKERS and NC and a reduction in the lymphocytes count in relation to EX-SMOKERS and COPD (Figure 1D).

Lymphopenia is a described characteristic in smokers and is linked to worst health prognoses (Biondini et al., 2019). Therefore, smoking may generate immunosuppressive effects, with a reduction in the number of lymphocytes (Düvenci Birben et al., 2016; Tulgar et al., 2016), and consequently a reduction in the anti-SARS-CoV-2 immune response. 
TABLE 1 | Patients' characteristics by on the hospitalization day.

\begin{tabular}{|c|c|c|c|c|c|c|c|c|c|c|}
\hline & \multicolumn{2}{|c|}{$N C(N=10)$} & \multicolumn{2}{|c|}{ EX-SMOKERS $(N=12)$} & \multicolumn{2}{|c|}{ SMOKERS $(N=7)$} & \multicolumn{2}{|c|}{ COPD $(N=6)$} & \multirow[b]{2}{*}{ Reference numbers } & \multirow[b]{2}{*}{$p$-value } \\
\hline & Mean & SEM & Mean & SEM & Mean & SEM & Mean & SEM & & \\
\hline Age (years) & 56.87 & 3.101 & 58.43 & 5.327 & 60.67 & 2.909 & 63.33 & 3.648 & - & 0.6657 \\
\hline Hospitalization time (days) & $18.3^{\star}$ & 2.856 & $37.42^{\star}$ & 4.94 & 32.29 & 9.411 & 31.17 & 5.924 & - & 0.0303 \\
\hline Lactate dehydrogenase (U/L) & 439 & 50.88 & 482.9 & 59.83 & 580.2 & 116.4 & 522.8 & 89.93 & $135-225$ & 0.5546 \\
\hline C-reactive protein (mg/L) & 184.5 & 43.14 & 143.7 & 45.9 & 191 & 68.06 & 116.7 & 37.54 & $<5.0$ & 0.7150 \\
\hline Alanine aminotransferase (U/L) & 69.5 & 24.78 & 54.25 & 14 & 35.57 & 8.893 & 47.5 & 13.4 & $<41$ & 0.4593 \\
\hline Aspartate aminotransferase (U/L) & 67.38 & 20.61 & 52.58 & 11.41 & 45.14 & 10.48 & 76.5 & 26.07 & $<37$ & 0.9193 \\
\hline D-dimer (ng/mL) & 1364 & 307 & 4745 & 2011 & 6042 & 3195 & 3604 & 2286 & $<500$ & 0.7710 \\
\hline Alkaline phosphatase (U/L) & 221.9 & 47.04 & 185.3 & 51.18 & 128.8 & 30.97 & 202.7 & 42.49 & $40-129$ & 0.3862 \\
\hline
\end{tabular}

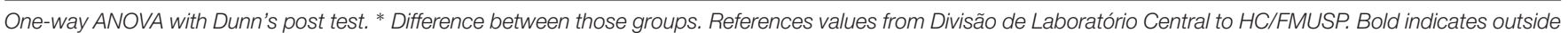

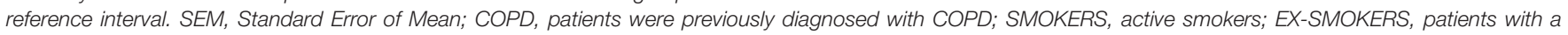
previous smoking history (with over 10 years absence) without COPD; and NC, healthy individuals non-smokers.

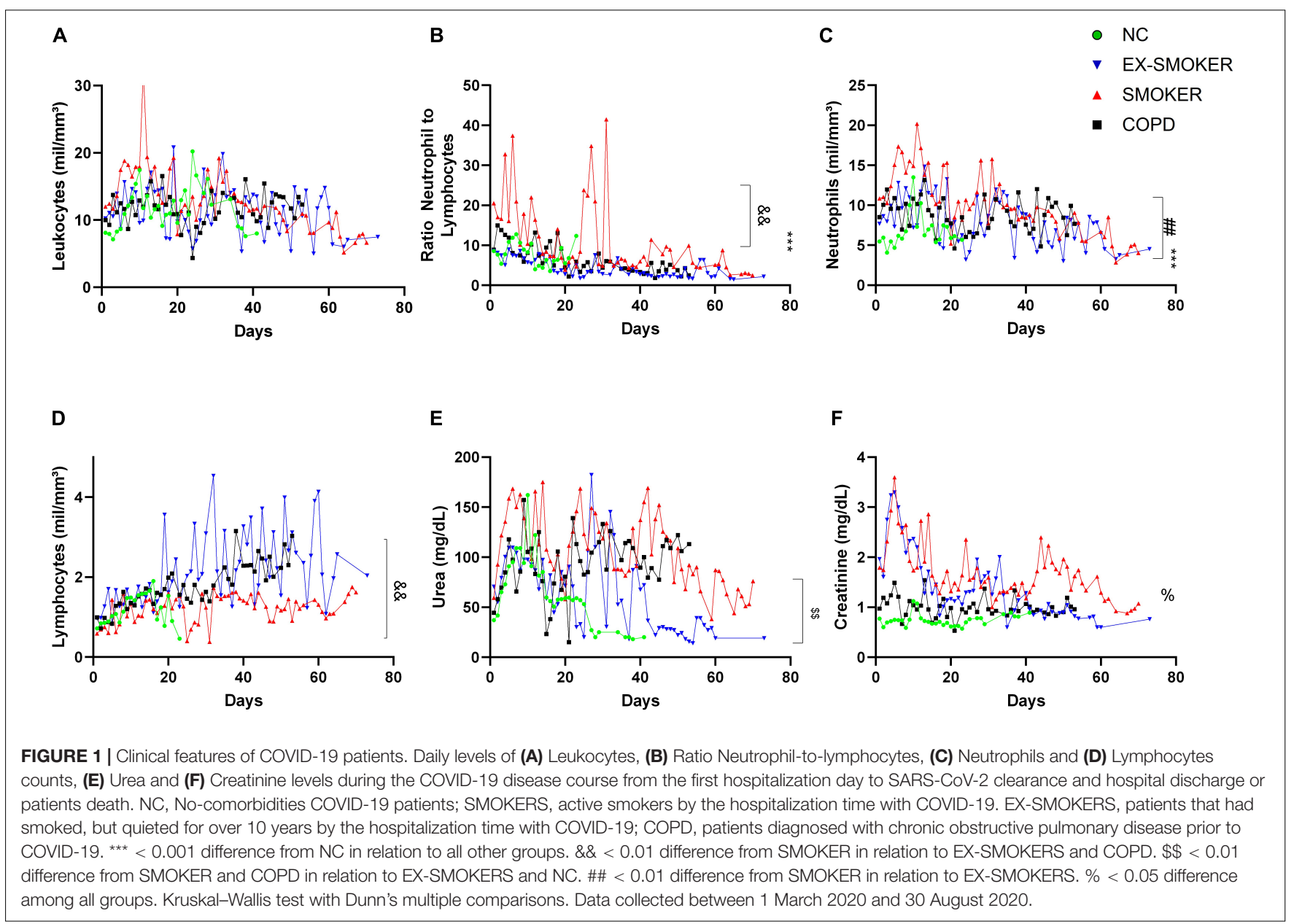

In our cohort, COPD and SMOKERS presented increased levels of urea in relation to $\mathrm{NC}$ and EX-SMOKERS (Figure 1E). Creatinine levels were different among all groups, indicating that EX-SMOKERS also presented an increase in this inflammatory marker in relation to NC group (Figure 1F). Alterations in the urea and creatinine levels among COPD and Smokers patients could also further indicate an increase in the risk of kidney injury during COVID-19 (Yang et al., 2020).

A report from Wong et al. identified that former smokers present an increased influenza-associated hazard ratio in comparison to non-smokers (Wong et al., 2013). Similarly, in our cohort, the group of EX-SMOKERS presented a difference in the disease course compared to the NC group. 
It's noteworthy that during the investigation two patients from the SMOKERS group passed away due to severe respiratory distress, corroborating with previous reports that identified that smokers with COVID-19 possess a higher odd of developing severe COVID-19. All other patients cleared SARS-CoV-2 infection and were discharged from the hospital. Therefore, in our cohort SMOKERS groups presented a statistically significant difference in the survival curve in comparison to all other groups $(p=0.0349)$.

This phenomenon in smokers and COPD patients could be partially explained by the local inflammatory and oxidative response (Tian et al., 2017) and the up-regulation of ACE2 receptor, SARS-CoV-2 entry receptor, in the lungs (Jacobs et al., 2020). In comparison, allergic asthma downregulates ACE2 receptor expression in the lungs (Castelo Branco et al., 2020), possibly being a protective factor in COVID-19 (Alberca et al., 2020e). This still needs to be further explored since the elderly, an established risk group for severe COVID-19, also presents a downregulation in the ACE2 receptor expression in the lungs (Tavares et al., 2020).

Our report highlights the difference in disease course among smokers, ex-smokers, and no smokers (NC group), indicating that ex-smokers do present a better disease course than smokers. Importantly, we demonstrate that COPD and smoking do influence COVID-19 disease course independently of other comorbidities. Is important to highlight that this investigation possesses a small number of patients and should be further expanded to better understand the influence of these comorbidities on COVID-19. A possible synergic effect of smoking and COPD with other comorbidities in COVID-19 needs to be further explored to aid in the development of specific treatments for these populations.

\section{CONCLUSION}

In our investigation on the hospitalization day non-smokers, smokers, former smokers, and COPD patients did not present

\section{REFERENCES}

Aikphaibul, P., Theerawit, T., Sophonphan, J., Wacharachaisurapol, N., Jitrungruengnij, N., and Puthanakit, T. (2020). Risk factors of severe hospitalized respiratory syncytial virus infection in tertiary care center in Thailand. Influenza Other Respi Viruses 5, 64-71. doi: 10.1111/irv.12793

Alberca, R., Aoki, V., and Sato, M. (2020a). COVID-19 and HIV: case reports of 2 co-infected patients with different disease courses. World Acad. Sci. J. 3:4. doi: 10.3892/wasj.2020.75

Alberca, R. W., Andrade, M. M., de, S., Castelo Branco, A. C. C., Pietrobon, A. J., Pereira, N. Z., et al. (2020b). Frequencies of CD33+ CD11b+ HLA-DR- CD14CD66b + and CD33+ CD11b+ HLA-DR- CD14+ CD66b- cells in peripheral blood as severity immune biomarkers in COVID-19. Front. Med. 7:580677. doi: 10.3389/FMED.2020.580677

Alberca, R. W., Oliveira, L., de, M., Branco, A. C. C. C., Pereira, N. Z., and Sato, M. N. (2020c). Obesity as a risk factor for COVID-19: an overview. Crit. Rev. Food Sci. Nutr. 15, 1-15. doi: 10.1080/10408398.2020.1775546

Alberca, R. W., Pereira, N. Z., Oliveira, L. M. D. S., Gozzi-Silva, S. C., and Sato, M. N. (2020d). Pregnancy, viral infection, and COVID-19. Front. Immunol. 11:1672. doi: 10.3389/FIMMU.2020.01672 differences in COVID-19 associated inflammatory markers. Nevertheless, a longitudinal investigation demonstrated that smokers and COPD patients, without other comorbidities, present a higher risk for severe COVID-19.

\section{DATA AVAILABILITY STATEMENT}

The original contributions presented in the study are included in the article/supplementary material, further inquiries can be directed to the corresponding author/s.

\section{ETHICS STATEMENT}

The studies involving human participants were reviewed and approved by the Ethics Committee of Hospital das Clinicas da Universidade de São Paulo - HCFMUSP (no. 30800520.7.0000.0068-2020). Written informed consent for participation was not required for this study in accordance with the National Legislation and the Institutional Requirements.

\section{AUTHOR CONTRIBUTIONS}

RA, GB, AD, and MS: conception, analyze the data, and write and review of the manuscript. JL, EO, SG-S, YR, MA, DB, LO, AB, AP, NP, FT, and IF: data collection, analyze the data, and review of the manuscript. All authors contributed to the article and approved the submitted version.

\section{FUNDING}

This work was supported by the Fundação de Amparo a Pesquisa do Estado de São Paulo (FAPESP) grants: 19/02679-7 and 17/18199-9 and Coordenação de Aperfeiçoamento de Pessoal de Nível Superior - CAPES: 88887.503842/2020-00.

Alberca, R. W., Yendo, T., Aoki, V., and Sato, M. N. (2020e). Asthmatic patients and COVID-19: different disease course? Allergy 1-2. doi: 10.1111/all.14601

Alberca, R. W., Yendo, T. M., Leuzzi Ramos, Y. Á, Fernandes, I. G., Oliveira, L. M., Teixeira, F. M. E., et al. (2020f). Case report: COVID-19 and chagas disease in two coinfected patients. Am. J. Trop. Med. Hyg. 103, 2353-2356.

Alberca, R. W. (2020). Asthma endotypes and COVID-19. J. Asthma 1, 1-2. doi: 10.1080/02770903.2020.1825731

Auvinen, R., Nohynek, H., Syrjänen, R., Ollgren, J., Kerttula, T., Mäntylä, J., et al. (2020). Comparison of the clinical characteristics and outcomes of hospitalized adult COVID-19 and influenza patients - a prospective observational study. Infect. Dis. (Auckl) 10, 1-11. doi: 10.1080/23744235.2020.18 40623

Barrasa, H., Rello, J., Tejada, S., Martín, A., Balziskueta, G., Vinuesa, C., et al. (2020). SARS-CoV-2 in spanish intensive care units: early experience with 15day survival in Vitoria. Anaesth Crit. Care Pain. Med. 39, 553-561. doi: 10.1016/ j.accpm.2020.04.001

Biondini, D., Semenzato, U., Bonato, M., Tinè, M., Bazzan, E., Damin, M., et al. (2019). Lymphopenia is linked to an increased incidence of cancer in smokers without COPD. Eur. Respiratory J. 54:A2586. doi: 10.1183/13993003.congress2019.pa2586 
Castelo Branco, A. C. C., Sato, M. N., and Alberca, R. W. (2020). The possible dual role of the ACE2 receptor in asthma and SARS-COV2 infection. Front. Cell Infect. Microbiol. 10:550571. doi: 10.3389/FCIMB.2020.550571

Codo, A. C., Davanzo, G. G., Monteiro, L. B., Souza, G., Muraro, S., Carregari, V., et al. (2020). Elevated glucose levels favor Sars-Cov-2 infection and monocyte response through a Hif- $1 \alpha /$ Glycolysis dependent axis. SSRN Electron. J. doi: $10.2139 /$ ssrn.3606770

Dai, M., Tao, L., Chen, Z., Tian, Z., Guo, X., Allen-Gipson, D. S., et al. (2020). Influence of cigarettes and alcohol on the severity and death of COVID-19: a multicenter retrospective study in Wuhan. China. Front. Physiol. 11:588553. doi: 10.3389/FPHYS.2020.588553

Düvenci Birben, Ö, Akçay, Ş, Sezer, S., Şirvan, Ş, and Haberal, M. (2016). Effect of smoking on peripheral blood lymphocyte subsets of patients with chronic renal failure. Exp. Clin. Transplant 14, 91-94. doi: 10.6002/ect.tondtdtd 2016.P34

Gilca, R., de Serres, G., Boulianne, N., Ouhoummane, N., Papenburg, J., DouvilleFradet, M., et al. (2011). Risk factors for hospitalization and severe outcomes of 2009 pandemic H1N1 influenza in Quebec, Canada. Influenza Other Respi Viruses 5, 247-255. doi: 10.1111/j.1750-2659.2011.00204.x

Guan, W., Ni, Z., Hu, Y., Liang, W., Ou, C., He, J., et al. (2020). Clinical characteristics of coronavirus disease 2019 in China. N. Engl. J. Med. 382, 1708-1720. doi: 10.1056/NEJMoa2002032

Han, H., Huang, W., Du, W., Shen, Q., Yang, Z., Li, M. D., et al. (2019). Involvement of interferon regulatory factor 7 in nicotine's suppression of antiviral immune responses. J. Neuroimmune Pharmacol. 14, 551-564. doi: 10.1007/s11481-01909845-2

Jacobs, M., van Eeckhoutte, H. P., Wijnant, S. R. A., Janssens, W., Brusselle, G. G., Joos, G. F., et al. (2020). Increased expression of ACE2, the SARS-CoV-2 entry receptor, in alveolar and bronchial epithelium of smokers and COPD subjects. Eur. Respir. J. 56:2002378. doi: 10.1183/13993003.02378-2020

Leung, J. M., Yang, C. X., Tam, A., Shaipanich, T., Hackett, T. L., Singhera, G. K., et al. (2020). ACE-2 expression in the small airway epithelia of smokers and COPD patients: implications for COVID-19. Eur. Respir. J. 55:2000688. doi: 10.1183/13993003.00688-2020

Lian, J., Jin, X., Hao, S., Jia, H., Cai, H., Zhang, X., et al. (2020). Epidemiological, clinical, and virological characteristics of 465 hospitalized cases of coronavirus disease 2019 (COVID-19) from Zhejiang province in China. Influenza Other Respi Viruses 14, 564-574. doi: 10.1111/irv.12758

Marsh, S., Aldington, S., Shirtchiffe, P., Weatherall, M., and Beasley, R. (2006). Smoking and COPD: what really are the risks? Eur. Respir. J. 28, 883-884. doi: 10.1183/09031936.06.00074806

Patanavanich, R., and Glantz, S. A. (2020). Smoking is associated with COVID-19 progression: a meta-analysis. Nicotine Tob Res. 22, 1653-1656. doi: 10.1093/ntr/ ntaa082

Shaw, P. D., Rao, A. P., and Malik, P. (2020). Cardiac complications of COVID-19: the concealed realities. Infect. Dis. (Auckl) 9, 1-2. doi: 10.1080/23744235.2020. 1841908

Singanayagam, A., Loo, S. L., Calderazzo, M., Finney, L. J., Torralbo, M. B. T., Bakhsoliani, E., et al. (2019). Antiviral immunity is impaired in COPD patients with frequent exacerbations. Am. J. Physiol. - Lung Cell Mol. Physiol. 317, L893-L903. doi: 10.1152/ajplung.00253.2019

Tavares, C., de Am, Avelino-Silva, T. J., Benard, G., Cardozo, F. A. M., Fernandes, J. R., et al. (2020). Ace2 expression and risk factors for covid-19 severity in patients with advanced age. Arq. Bras. Cardiol. 115, 701-707. doi: 10.36660/ abc. 20200487

Tian, Z., Zhang, H., DIxon, J., Traphagen, N., Wyatt, T. A., Kharbanda, K., et al. (2017). Cigarette smoke impairs A 2A adenosine receptor mediated wound repair through up-regulation of Duox-1 expression. Sci. Rep. 7:44405. doi: $10.1038 /$ srep44405

Tulgar, Y. K., Cakar, S., Tulgar, S., Dalkilic, O., Cakiroglu, B., and Uyanik, B. S. (2016). The effect of smoking on neutrophil/ lymphocyte and platelet/lymphocyte ratio and platelet indices: a retrospective study. Eur. Rev. Med. Pharmacol. Sci. 20, 3112-3118.

Vardavas, C. I., and Nikitara, K. (2020). COVID-19 and smoking: a systematic review of the evidence. Tob Induc. Dis. 18:20. doi: 10.18332/tid/119324

Wolff, D., Nee, S., Hickey, N. S., and Marschollek, M. (2020). Risk factors for Covid-19 severity and fatality: a structured literature review. Infection 28, 1-14. doi: 10.1007/s15010-020-01509-1

Wong, C. M., Yang, L., Chan, K. P., Chan, W. M., Song, L., Lai, H. K., et al. (2013). Cigarette smoking as a risk factor for influenza-associated mortality: evidence from an elderly cohort. Influenza Other Respi Viruses 7, 531-539. doi: 10.1111/j.1750-2659.2012.00411.x

Yang, X., Jin, Y., Li, R., Zhang, Z., Sun, R., and Chen, D. (2020). Prevalence and impact of acute renal impairment on COVID-19: a systematic review and meta-analysis. Crit. Care 24:356. doi: 10.1186/s13054-020-03065-4

Yildiz, H., Yombi, J. C., and Castanares-Zapatero, D. (2020). Validation of a risk score to predict patients at risk of critical illness with COVID-19. Infect. Dis. (Auckl) 1, 1-3. doi: 10.1080/23744235.2020.1823469

Yoon, J. G., Noh, J. Y., Choi, W. S., Lee, J., Lee, J. S., Wie, S. H., et al. (2020). A comparison of epidemiology and clinical outcomes between influenza a H1N1pdm09 and H3N2 based on multicenter surveillance from 2014 to 2018 in South Korea. Influenza Other Respi Viruses 15, 99-109. doi: 10.1111/irv.12795

Zhao, Q., Meng, M., Kumar, R., Wu, Y., Huang, J., Lian, N., et al. (2020). The impact of COPD and smoking history on the severity of Covid-19: a systemic review and meta-analysis. J. Med. Virol. 92, 1915-1921. doi: 10.1002/jmv.25889

Conflict of Interest: The authors declare that the research was conducted in the absence of any commercial or financial relationships that could be construed as a potential conflict of interest.

Copyright (c) 2021 Alberca, Lima, Oliveira, Gozzi-Silva, Ramos, Andrade, Beserra, Oliveira, Branco, Pietrobon, Pereira, Teixeira, Fernandes, Duarte, Benard and Sato. This is an open-access article distributed under the terms of the Creative Commons Attribution License (CC BY). The use, distribution or reproduction in other forums is permitted, provided the original author(s) and the copyright owner(s) are credited and that the original publication in this journal is cited, in accordance with accepted academic practice. No use, distribution or reproduction is permitted which does not comply with these terms. 